\section{Hier steht eine Anzeige.}

Springer

\title{
Schlaganfallprävention bei Vorhofflimmern
}

\section{Was bringt der Verschluss des linken Vorhofohrs?}

Fragestellung: Ist der Vorhofohrverschluss bei Patienten mit Vorhofflimmern eine Alternative zu einer oralen Antikoagulation mit Warfarin?

Hintergrund: Der interventionelle Vorhofohrverschluss ist eine potenziell wirksame Methode um bei Patienten mit Vorhofflimmern die Bildung von Thromben im Vorhofohr zu verhindern und damit das Schlaganfallrisiko zu reduzieren. Die beiden randomisierten Studien, in denen diese Methode mit einer oralen Antikoagulation mit VKA verglichen wurde, zeigten allerdings keine Überlegenheit der Intervention gegenüber einer antithrombotischen Therapie $[1,2]$.

Patienten und Methodik: In die Metaanalyse wurden 707 Patienten aus der PROTECT-AF-Studie eingeschlossen, 407 aus der PREVAIL-Studie, 566 aus dem CAPRegister und 579 aus dem CAP2-Register. Als Endpunkte wurden unter anderem Schlaganfälle, systemische Embolien und kardiovaskulärer Tod erfasst.

Ergebnisse: Die Metaanalyse umfasste 2.406 Patienten mit 5.931 Patientenjahren (mittlere Beobachtungszeit 2,7 Jahre). Die meisten Patienten hatten einen $\mathrm{CHADS}_{2}$ Score von über 3. Bezüglich des primären Endpunkts (Schlaganfall, systemische Embolie und kardiovaskulärer Tod) bestand kein Unterschied zwischen den beiden Behandlungsformen (Vorhofohrverschluss: 2,72\% pro Jahr, Warfarin: 3,5\% pro Jahr). Ischämische Schlaganfälle waren mit $1,6 \%$ versus $0,9 \%$ in der Interventionsgruppe signifikant häufiger. Zerebrale Blutungen waren mit 0,15\% versus $0,96 \%$ in der Gruppe mit Vorhofohrverschluss signifikant reduziert.

Schlussfolgerungen: In einer Metaanalyse von zwei randomisierten Studien und zwei großen

Holmes DR Jr, Doshi SK, Kar S et al. Left atrial appendage closure as an alternative to warfarin for stroke prevention in atrial fibrillation: A patient-level metaanalysis. J Am Coll Cardiol 2015; 65: $2614-23$ Registern führte der Vorhofohrverschluss bei Patienten mit Vorhofflimmern im Vergleich zu einer oralen Antikoagulation mit Warfarin nicht zu einer Reduktion des kombinierten Endpunkts. Ischämische Insulte waren in der Interventionsgruppe häufiger, zerebrale Blutungen in der Antikoagulationsgruppe.

\section{- Kommentar von Hans-Christoph Diener, Essen}

\section{Daten zu den „idealen“ Kandidaten fehlen}

Ein prinzipielles Problem der bisher durchgeführten Studien war, dass in diese nicht die Patienten eingeschlossen wurden, die eigentlich ideale Kandidaten für einen Vorhofohrverschluss sind, nämlich Patienten mit einem hohen Langzeitrisiko für Blutungskomplikationen unter oraler Antikoagulation, denen man zumindest eine kurzfristige Antikoagulation nach Implantation des Devices oder eine duale Plättchenhemmung zumuten könnte. Daten, die den Vorhofohrverschluss mit neuen Antikoagulanzien vergleichen, die eine deutlich geringere Rate an intrazerebralen Blutungen haben, liegen ebenfalls nicht vor.

\footnotetext{
Referenzen:

1. Reddy VY et al. JAMA 2014; 312: $1988-98$

2. Reddy VY et al. Circulation 2013; 127: 720 - 9
} 